5. Driving-clock errors and irregularities are not 兽magnified in the Schaeberle tower camera; such errors ind irregularities in a large cœlostatic camera are inagnified greatly on the sensitive film.

6. The lens in the Schaeberle tower camera is colInimated for the Sun at mid-totality; but the lack of , collimation at the beginning and ending of totality 'óppears to be negligible even for eclipses of long duraI- Ition, with the lens of aperture 5 inches, and focal length 480 inches-ratio 1:96.

7. It is probably as easy to protect the inner tower as the cœlostat against wind vibrations, remembering that colostat disturbances are highly magnified in the long-focus image.

Six exposures were made with the 40-foot camera, on Seed's plates, No. 27, as below:

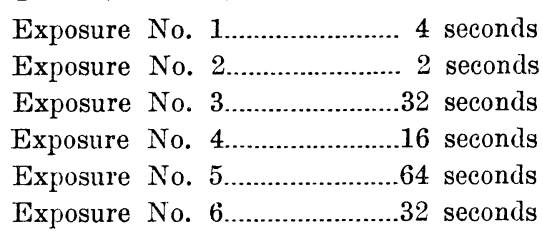

Returning sunlight fell on the last plate for a fraction of a second. The seeing was splendid, the focus and clock-driving were perfect, and the negatives are excellent. The diameter of the Moon's image on the original negatives is 4.74 inches. Two of the negatives have "standard squares" near one edge, made by exposing the squares to the light of a Hefner amyl-acetate lamp on the night preceding the eclipse.

The first and last exposures recorded the chromospheric stratum. There were several short prominences on the east limb, several larger ones on the west limb, and a large eruptive prominence in position angle $214^{\circ}$. The plates with shorter exposures show a great number of short narrow streamers, especially in the polar regions. The longer exposures recorded about thirty well defined long streamers, one-half of them extending out a distance of one lunar diameter and the other half a distance equal to one and a half lunar diameters. The inner corona on the east side of the Sun is very irregular in structure, and there is a disturbed region, apparently nearly conical in form, similar to those observed on the Crocker photographs of the Sumatra (1901) and Spain (1905) eclipses. The radiating streamers appear as if they diverge from the vertex of the cone situated in position angle $75^{\circ} \pm$, and at distance from Sun's center $12^{\prime} \pm$. A prominence near position $80^{\circ}$ appears like the top of an eruptive prominence which may or may not have its base near the vertex described. An interesting streamer, starting near the south point, and extending, not radially, but in direction $190^{\circ}$, is unusually straight and slender and falls off very slowly in intensity. At a distance of $1 / 3$ diameter from the limb a fainter streamer branches from it, the two making an angle of $4^{\circ}$ with each other. Both are recorded to a distance of $1 \frac{1}{2}$ diameters.

All the observers with previous eclipse experience noted from their visual observations that the outer corona was relatively fainter than at former eclipses. The large-scale photographs confirm this view. It is probably a safe estimate that less than one-thousandth part of the coronal light came from a distance greater than 6 or 7 minutes of arc from the Sun's limb. On the negative with $64^{\mathrm{s}}$ exposure, the fully exposed ring stops quite abruptly at the distance stated.

W. W. Campbell,
Sebastian Albrecht.

\title{
PHOTOMETRIC OBSERVATIONS OF THE CORONA.
}

An attempt was made to determine the photographic brightness of the corona with apparatus similar to that used in Spain at the 1905 eclipse. Instead, however, of comparing the corona directly with the Moon, as was done in 1905 , standard squares were impressed on the plates of 1908 by means of a Hefner amyl-acetate lamp. Comparisons with the Moon or other sources will be made later, through the same lamp.

Two plates were exposed to the light of the corona, when the sky was entirely clear of clouds. The resulting photographs are excellent and will give good values of the total effective brightness of the corona when it becomes possible to compare the intensities with suitable apparatus.

These plates contain the data, also, for a good determination of the brightness of the sky immediately . about the corona.

The preliminary examination of the plates shows that the effective photographic light came alrnost wholly from the extreme inner corona, within $1^{\prime}$ or $2^{\prime}$ of the Sun's surface.

C. D. Perrine.

F'ebruary $3,1908$. 